\title{
Comparative Evaluation of the Biodistribution Profiles of a Series of Nonpeptidic Neurotensin Receptor-1 Antagonists Reveals a Promising Candidate for Theranostic Applications
}

\author{
Jörg Schulz*1, Martin Rohracker*1, Marvin Stiebler ${ }^{1}$, Jürgen Goldschmidt ${ }^{2}$, Oliver S. Grosser ${ }^{1}$, Frank Osterkamp ${ }^{3}$, \\ Annette Pethe ${ }^{1}$, Ulrich Reineke ${ }^{3}$, Christiane Smerling ${ }^{3}$, and Holger Amthauer ${ }^{1,4}$ \\ ${ }^{I}$ Klinik für Radiologie und Nuklearmedizin, Otto-von-Guericke Universität Magdeburg, Magdeburg, Germany; ${ }^{2}$ Leibniz-Institut für \\ Neurobiologie, Magdeburg, Germany; 33 B Pharmaceuticals GmbH, Berlin, Germany; and ${ }^{4}$ Department of Nuclear Medicine, \\ Charité-Universitätsmedizin, Berlin, Germany
}

\begin{abstract}
Neurotensin receptor-1 (NTR1) is a promising target for diagnostic imaging and targeted radionuclide therapy. The aim of this study was to evaluate the biodistribution profiles of a series of newly developed diarylpyrazole-based NTR1 antagonists regarding their suitability as diagnostic and potentially radiotherapeutic agents. Methods: 3BP-227, 3BP-228, and 3BP-483 were labeled with ${ }^{111}$ In and injected intravenously into NTR1-positive HT29 xenograftbearing nude mice. At 3, 6, 12, and $24 \mathrm{~h}$ after administration, SPECT/ CT images were acquired or mice were sacrificed for ex vivo determination of tissue-associated radioactivity. Results: High-contrast tumor visualization in SPECT/CT images was achieved using the 3 compounds of this study. Ex vivo biodistribution studies confirmed a high and persistent tumor uptake, peaking at $6 \mathrm{~h}$ after injection for ${ }^{111} \mathrm{In}-3 \mathrm{BP}-227(8.4 \pm 3.1$ percentage injected dose per gram [\% ID/g]) and at $3 \mathrm{~h}$ after injection for ${ }^{111} \mathrm{In}-3 \mathrm{BP}-228(10.2 \pm 5.3 \% \mathrm{ID} / \mathrm{g})$ and ${ }^{111} \mathrm{In}-3 \mathrm{BP}-483(1.9 \pm 0.8 \% \mathrm{ID} / \mathrm{g})$. Tumor-to-normal-tissue ratios obtained with ${ }^{111} \mathrm{In}-3 \mathrm{BP}-227$ and ${ }^{111} \mathrm{In}-3 \mathrm{BP}-228$ were consistently greater than 1. Conclusion: On the basis of the superior biodistribution profile compared with previously reported radiolabeled NTR1 ligands, ${ }^{111}$ In-3BP-227 is an ideal candidate for further development as a theranostic tracer.
\end{abstract}

Key Words: NTR1-radiotracer; xenograft; SPECT/CT; biodistribution; oncology

J Nucl Med 2016; 57:1120-1123

DOI: 10.2967/jnumed.115.170530

C ertain peptide receptors, such as the neurotensin receptors (NTRs), are commonly overexpressed in many primary human tumors and their metastases, making them ideal targets for molecular imaging and targeted cancer therapy $(1,2)$. The NTR subtype 1 (NTR1) has been associated with several oncogenic effects such as proliferation, survival, migration, invasion, and neoangiogenesis $(3,4)$ and has been demonstrated to be overexpressed, inter alia,

Received Nov. 30, 2015; revision accepted Feb. 10, 2016.

For correspondence or reprints contact: Holger Amthauer, Klinik für Radiologie und Nuklearmedizin, Otto-von-Guericke Universität, Leipziger Strasse 44, 39120 Magdeburg, Germany.

E-mail: holger.amthauer@med.ovgu.de

${ }^{*}$ Contributed equally to this work.

Published online Mar. 3, 2016.

COPYRIGHT (c) 2016 by the Society of Nuclear Medicine and Molecular Imaging, Inc. in ductal pancreatic cancer, Ewing's sarcoma, and small cell lung cancer (1). Hence, NTR1 imaging might be a promising tool for tumor diagnosis and staging.

Previous attempts at specific delivery of diagnostic and therapeutic radionuclides to tumors using NTR1 as a target have focused on the development of radiolabeled derivatives of its natural ligand neurotensin with improved metabolic stability (5). Although some of the reported radiolabeled agonistic peptides achieved a distinct tumor uptake, in the cases of ${ }^{177} \mathrm{Lu}$ or ${ }^{188} \mathrm{Re}$ payloads even accompanied by a certain antitumoral effect, overall results were compromised by varying degrees of relatively low and unfavorable tumor-to-background ratios. Because recent developments in somatostatin and gastrin-releasing peptide receptor targeting indicate a superior tumor uptake and retention of radiolabeled antagonists compared with agonists (6-8), Osterkamp et al. modified the known NTR1 antagonist SR142948A $(9,10)$ by attaching short linker-chelator moieties, yielding the novel diarylpyrazole-based NTR1 antagonists 3BP-227, -228, and -483 (Fig. 1). The incorporated DOTA chelator is capable of forming stable complexes with a wide range of diagnostic (e.g., ${ }^{111} \mathrm{In}$ or ${ }^{68} \mathrm{Ga}$ ) and therapeutic (e.g., ${ }^{177} \mathrm{Lu}$ or ${ }^{90} \mathrm{Y}$ ) radionuclides (11). In vitro evaluation of the compounds indicated a high NTR1 affinity and specificity as well as adequate metabolic stability in human and murine plasma, which warranted the further examination in in vivo studies. The aim of this study was to evaluate and compare the potential for clinical translation of 3BP-227, 3BP-228, and 3BP-483 based on their imaging performance and biodistribution profiles in tumor-bearing mice.

\section{MATERIALS AND METHODS}

\section{Radiolabeling}

Compounds were dissolved in buffer ( $0.4 \mathrm{M}$ acetate, $0.325 \mathrm{M}$ gentisic acid, pH 5), and aliquots (3BP-227, 4.4-39.0 $\mu \mathrm{g}$; 3BP-228, $9.0 \mu \mathrm{g}$; 3BP$483,4.6-9.2 \mu \mathrm{g}$ ) were incubated with $200-400 \mathrm{MBq}$ of ${ }^{111} \mathrm{InCl}_{3}$ in $0.05 \mathrm{M} \mathrm{HCl}$ (Covidien) at $95^{\circ} \mathrm{C}$ for $30 \mathrm{~min}$. After being cooled, the product was analyzed by instant thin-layer and high-performance liquid chromatography and formulated in $0.9 \% \mathrm{NaCl}$ for intravenous administration. Detailed analytic methods can be found in the supplemental data (supplemental materials are available at http://jnm.snmjournals.org).

\section{Tumor Model}

Experiments were approved by the local animal welfare committee and performed according to national regulations. Mice were kept in individually ventilated cages under standard conditions with food and water ad libitum. NTR1-positive HT29 human colorectal carcinoma 


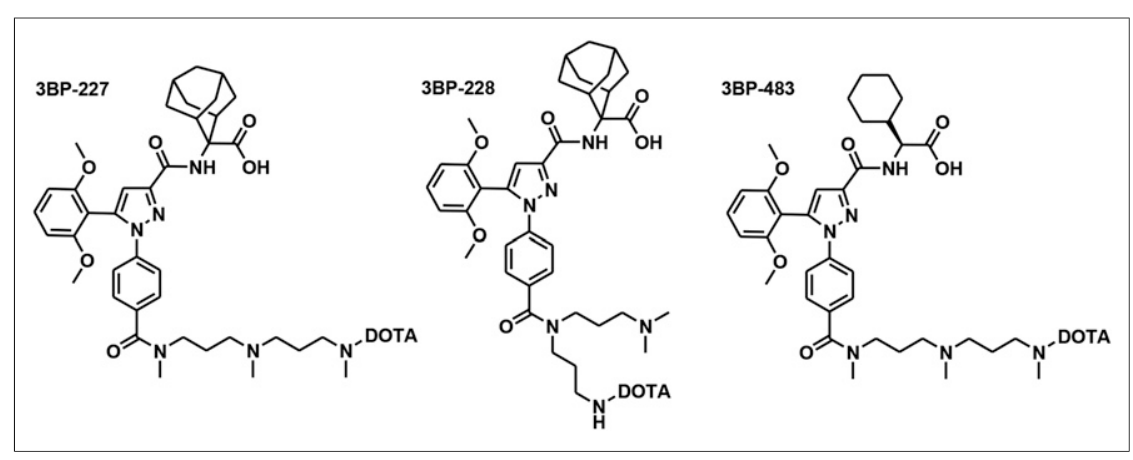

FIGURE 1. Chemical structures of 3BP-227, 3BP-228, and 3BP-483.

cells (European Collection of Authenticated Cell Cultures and Cell Lines Services) (12) and NTR1-negative HEK293 human embryonic kidney cells (European Collection of Authenticated Cell Cultures) (13) were grown in McCoy 5A modified medium (Biochrom) and Dulbecco modified Eagle medium, respectively, each supplemented with $10 \%$ fetal calf serum, $2 \mathrm{mM}$ L-glutamine, penicillin $(10,000 \mathrm{U} / \mathrm{mL})$, and streptomycin $(10 \mathrm{mg} / \mathrm{mL})$ at $37^{\circ} \mathrm{C}$ in $5 \% \mathrm{CO}_{2}$. Cells $\left(2-3 \times 10^{6}\right.$ suspended in McCoy $5 \mathrm{~A}$ modified medium, $40 \times 10^{6}$ cells $/ \mathrm{mL}$ ) were inoculated subcutaneously in the right flank of 6- to 8-wk-old athymic nu/nu female mice (NudeFoxn1 ${ }^{n u}$; Harlan Laboratories). Biodistribution and imaging studies were performed 2-4 wk after inoculation when the tumor was palpable.

\section{SPECT/CT Imaging}

Mice were injected via a lateral tail vein with ${ }^{111}$ In-labeled 3BP-227 $(21-47 \mathrm{MBq} ; n=6)$, 3BP-228 (33-44 MBq; $n=4)$, or 3BP-483 (31-44 $\mathrm{MBq} ; n=3)$ under isoflurane anesthesia $\left(1 \%-2 \%\right.$ isoflurane in $2: 1 \mathrm{O}_{2}$ : $\mathrm{N}_{2} \mathrm{O}$ volume ratio). Whole-body imaging was performed $3,6,12$, and $24 \mathrm{~h}$ after injection with a 4-head NanoSPECT/CT scanner (Mediso Ltd.). SPECT scans were acquired using a multipinhole collimator set (SCIVIS), each collimator with 9 pinholes (1.4-mm pinhole diameters). SPECT imaging was performed with 2 energy windows of $171 \mathrm{keV} \pm$ $5 \%$ and $245 \mathrm{keV} \pm 5 \%$ and an acquisition time of $60 \mathrm{~min}$. Images were reconstructed with isotropic voxel output sizes of 300-600 $\mu \mathrm{m}$, depending on counting rates (HiSPECT; SCIVIS). CT scans were acquired at a $192-\mu \mathrm{m}$ isotropic detector resolution and reconstructed at isotropic voxel sizes of $200 \mu \mathrm{m}$ (InVivoScope 1.43; Bioscan). After the last acquisition, mice were sacrificed and included into the 24-h-after-injection cohort for the ex vivo biodistribution studies described below.

\section{Ex Vivo Biodistribution Studies}

Mice were injected intravenously with ${ }^{111}$ In-labeled 3BP-227 (0.212.2 MBq), 3BP-228 (5.5-7.6 MBq), or 3BP-483 (4.9-18.4 MBq) under isoflurane anesthesia and sacrificed 3, 6, 12, or 24 h later. For each time point and tracer, 3-9 animals were examined. As a control for receptor specificity, mice bearing NTR1-negative HEK293-tumors $(n=3)$ were injected intravenously with ${ }^{111} \mathrm{In}-3 \mathrm{BP}-227$ and sacrificed $12 \mathrm{~h}$ after administration. Blood, urine, organs, and tumors were collected and weighed, and their activity content was assessed by wellcounter measurement. Tissue counts and injected dose for individual mice were decay-corrected to the time of euthanasia. Tissue uptake was expressed as the percentage injected dose per gram of tissue $(\% \mathrm{ID} / \mathrm{g})$.

\section{Data Analysis}

OsiriX MD (open source version 1.3, 64-bit) was used for image analysis. CT-guided delineation of volumes of interest for selected tissues was performed on the reconstructed SPECT dataset. Tumor-to-kidney activity ratios were calculated by dividing counting rates of the corresponding volumes of interest. The estimated ratios were correlated to tumor-to-kidney uptake ratios determined by ex vivo biodistribution using Spearman correlation.
Statistical analyses of differences in tissue uptake values and tumor-to-normal-tissue ratios were performed using ANOVA followed by the Tukey post hoc test for multiple comparisons and $t$ test for comparing 2 groups. Differences of $P$ values less than 0.05 were considered statistically significant.

\section{RESULTS}

\section{Radiolabeling}

Radiochemical yields of ${ }^{111}$ In labeling were $97 \%$ or greater for all compounds. The specific activity of ${ }^{111} \mathrm{In}-3 \mathrm{BP}-227$ was 4.4-6.7 MBq/nmol in initial experiments and increased to $26.0-66.8 \mathrm{MBq} / \mathrm{nmol}$ after further optimization. The specific activities of ${ }^{111} \mathrm{In}-3 \mathrm{BP}-228$ and ${ }^{111} \mathrm{In}-3 \mathrm{BP}-483$ were 34.6-35.7 and 33.3-62.8 MBq/nmol, respectively. Instant thin-layer and high-performance liquid chromatography profiles confirmed high radiochemical purity $(\geq 97 \%)$ as exemplified for ${ }^{111} \mathrm{In}-3 \mathrm{BP}-$ 227 in Figure 2.

\section{SPECT/CT Imaging}

High tumor-to-background contrast allowed the clear visualization of HT29 tumors by all $3{ }^{111}$ In-labeled compounds using SPECT/CT imaging (Fig. 3). Compared with ${ }^{111}$ In-3BP-483, ${ }^{111} \mathrm{In}-3 \mathrm{BP}-228$ and ${ }^{111}$ In-3BP-227 displayed higher tumor accumulation at all time points examined. ${ }^{111} \mathrm{In}-3 \mathrm{BP}-483$ also had the highest relative
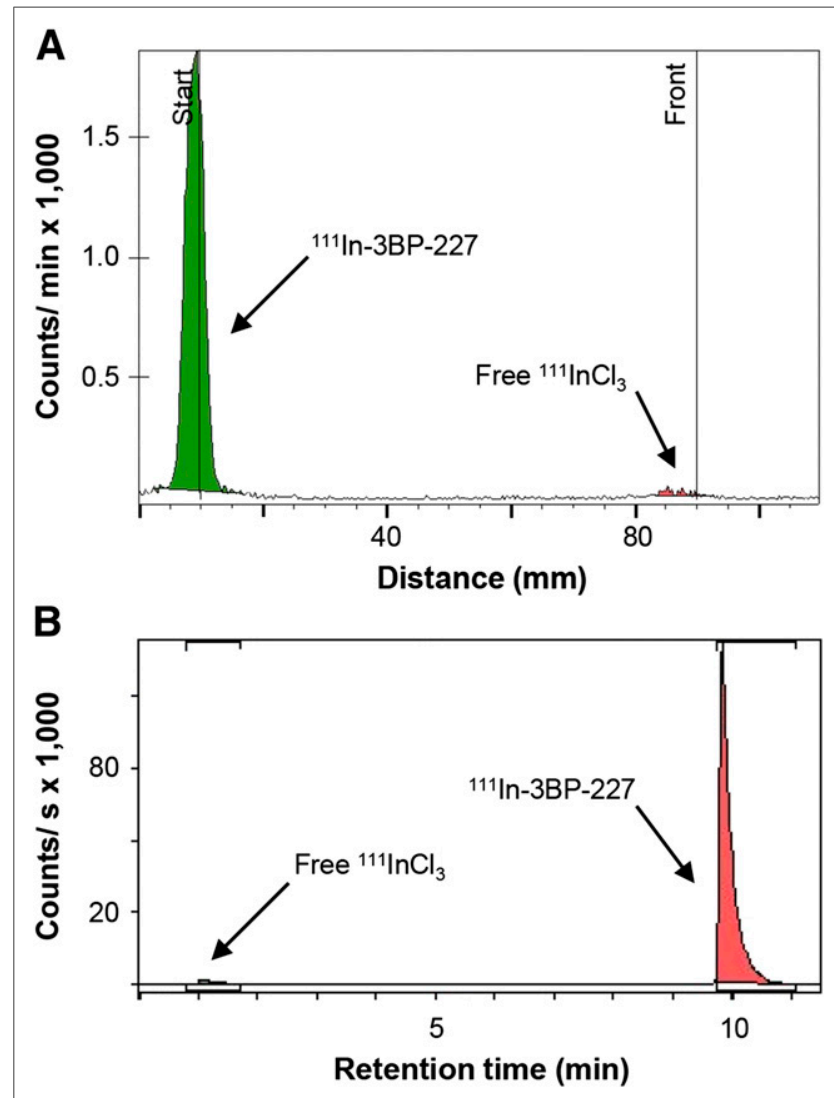

FIGURE 2. Thin-layer (A) and high-performance (B) liquid radiochromatograms after ${ }^{111}$ In labeling of 3BP-227, representative for each of the 3 compounds. 


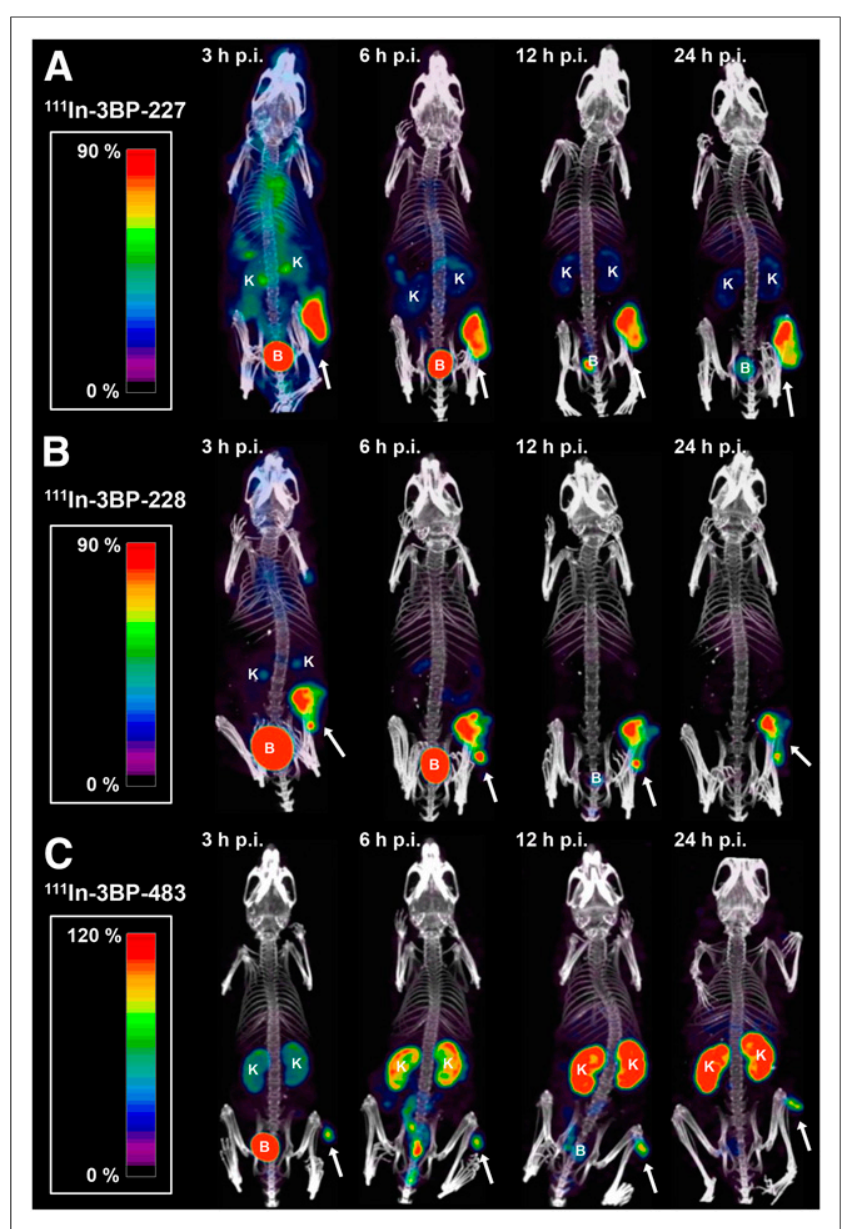

FIGURE 3. Representative images of 3 female nude mice bearing HT29 xenografts acquired by SPECT/CT at 3, 6, 12, and $24 \mathrm{~h}$ after injection (p.i.) of $22 \mathrm{MBq}$ of ${ }^{111} \mathrm{In}-3 \mathrm{BP}-227(\mathrm{~A}), 30 \mathrm{MBq}$ of ${ }^{111} \mathrm{In}-3 \mathrm{BP}-228(\mathrm{~B})$, and $31 \mathrm{MBq}$ of ${ }^{111}$ In-3BP-483 (C). Arrows show HT29. SPECT data were scaled to $0 \%-90 \%$ ( $A$ and $B$ ) or $0 \%-120 \%$ (C) of maximum count values in HT29 tumors in the respective datasets. $\mathrm{B}=$ urinary bladder; $\mathrm{K}=$ kidney.

kidney uptake, with tumor-to-kidney ratios decreasing from 0.9 at $3 \mathrm{~h}$ after injection to 0.5 at $24 \mathrm{~h}$ after injection. In contrast, tumor-tokidney ratios increased from 2.0 and 3.3 at $3 \mathrm{~h}$ after injection to 2.7 and 6.6 at $24 \mathrm{~h}$ after injection for ${ }^{111} \mathrm{In}-3 \mathrm{BP}-227$ and ${ }^{111} \mathrm{In}-3 \mathrm{BP}-228$, respectively.

\section{Ex Vivo Biodistribution Studies}

Ex vivo biodistribution data confirmed the high and specific tumor uptake of all 3 compounds (Fig. 4; Supplemental Tables 1-3). The uptake of ${ }^{111} \mathrm{In}-3 \mathrm{BP}-227$ in HT29 tumors peaked at $6 \mathrm{~h}$ after injection with $8.4 \pm 3.1 \% \mathrm{ID} / \mathrm{g}$, whereas maximum uptake for ${ }^{111} \mathrm{In}-3 \mathrm{BP}-228$ and -483 was observed at $3 \mathrm{~h}$ after injection with $10.2 \pm 5.2$ and $1.9 \pm 0.8 \% \mathrm{ID} / \mathrm{g}$, respectively. Tumor accumulation profiles of ${ }^{111} \mathrm{In}-3 \mathrm{BP}-227$ and ${ }^{111} \mathrm{In}-3 \mathrm{BP}-228$ were similar at 3, 6, and $12 \mathrm{~h}$ after injection, indicating high initial uptake followed by slow tumor activity washout. However, $24 \mathrm{~h}$ after administration, the tumor uptake of ${ }^{111} \mathrm{In}-3 \mathrm{BP}-227$ was significantly higher than that of ${ }^{111} \mathrm{In}-3 \mathrm{BP}-228(2.3 \pm 1.3$ vs. $0.8 \pm 0.2 \% \mathrm{ID} / \mathrm{g}, P=$ 0.020). For both compounds, tumor-to-normal-tissue ratios were greater than 1 at all times after injection, and tumor-to-kidney ratios were generally higher for ${ }^{111} \mathrm{In}-3 \mathrm{BP}-228$ than ${ }^{111} \mathrm{In}-3 \mathrm{BP}-227$. HT29 tumor uptake and tumor-to-background ratios of ${ }^{111} \mathrm{In}-3 \mathrm{BP}-483$ were significantly lower than for the other 2 compounds. A strong correlation of $R=0.97(P<0.001)$ between tumor-to-kidney ratios determined by imaging and ex vivo counting demonstrated the comparability of these 2 methods. ${ }^{111}$ In-3BP-227 uptake in NTR1-negative HEK293 xenografts was 11-fold lower than in HT29 tumors at $12 \mathrm{~h}$ after injection $(P=0.001$; Supplemental Table 1$)$.

\section{DISCUSSION}

Several preclinical studies and a few clinical studies have previously addressed NTR1 as a target for nuclear oncology $(5,6)$. Most of these studies focused on modifications of the natural peptide ligand neurotensin, which is labile toward enzymatic cleavage in vivo. However, attempts to stabilize analogs and improve properties for nuclear imaging and therapy have been of limited success (5). Although yielding good tumor localization in PET, the only nonpeptidic NTR1 antagonist used as a tracer to date does not allow labeling with therapeutic radionuclides such as ${ }^{177} \mathrm{Lu}$ because of its lack of a chelator moiety (14). We now report the novel and highly tumor-specific NTR1 antagonists 3BP-227, 3BP-228, and 3BP-483 for diagnostic imaging and potentially therapeutic applications in NTR1-positive tumors.

Despite their structural similarity, the 3 compounds displayed a distinct behavior in vivo. Uptake of ${ }^{111} \mathrm{In}-3 \mathrm{BP}-227$ and ${ }^{111} \mathrm{In}-3 \mathrm{BP}-$ 228 in the tumor exceeded that in all other tissues at all times after injection, a result that has not yet been achieved with NTR1 agonists. The biodistribution profiles of ${ }^{111} \mathrm{In}-3 \mathrm{BP}-227$ and ${ }^{111} \mathrm{In}-3 \mathrm{BP}-228$ were similar, with higher tumor-to-kidney ratios for ${ }^{111}$ In-3BP-228. However, ${ }^{111} \mathrm{In}-3 \mathrm{BP}-227$ tumor uptake was higher at $24 \mathrm{~h}$ after injection, indicating prolonged retention of ${ }^{111} \mathrm{In}-3 \mathrm{BP}-227$ in the target tissue, and a potential advantage when considering the suitability for targeted radiotherapy. Low uptake of ${ }^{111} \mathrm{In}-3 \mathrm{BP}-227$ in NTR1-negative

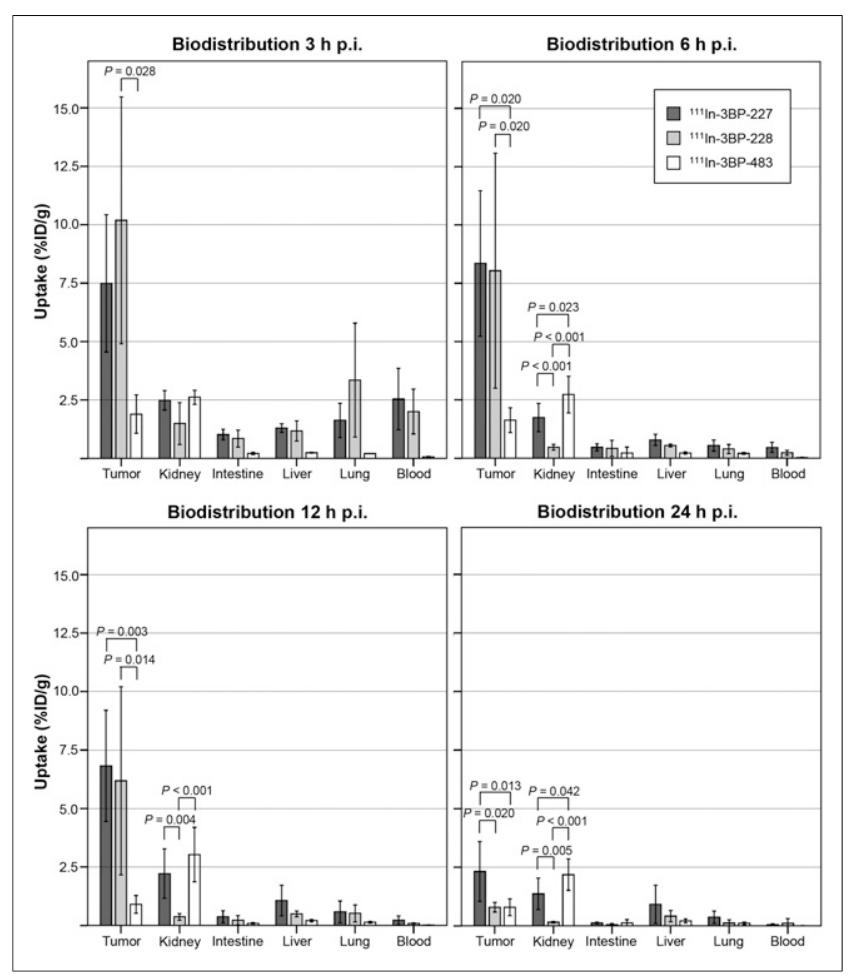

FIGURE 4. Uptake of ${ }^{111} \mathrm{In}-3 \mathrm{BP}-227,-228$, or -483 in selected tissues $3,6,12$, and $24 \mathrm{~h}$ after injection (p.i.) (mean $\% \mathrm{ID} / \mathrm{g} \pm \mathrm{SD}$ ). 
HEK tumors suggests high target specificity and low unspecific binding. ${ }^{111}$ In-3BP-483 exhibited the least favorable biodistribution profile, with tumor-to-kidney uptake ratios indicating a faster washout from tumor than from kidney tissue.

Interestingly, the differential tumor uptake of the 3 compounds, especially at the earlier time points (3-12 $\mathrm{h}$ after injection), parallels their potency in an in vitro functional calcium assay (half maximal inhibitory concentration for nat In-labeled compounds: 3BP-227, $5.35 \mathrm{nM}$; 3BP-228, $14.4 \mathrm{nM}$; 3BP-483, $75 \mathrm{nM}(11)$ ). Binding of agonistic ligands to NTR1 causes phospholipase C activation, which leads to an increase in inositol phosphate levels and calcium mobilization, whereas receptor antagonists inhibit neurotensin-stimulated calcium flux (9), with 3BP-227 and 3BP228 being particularly potent competitive inhibitors in our series. However, only the insights into the general biodistribution pattern with differential kidney accumulation and tumor-to-normal-tissue ratios obtained in this in vivo study allow conclusions about the potential suitability of each compound for radiotherapeutic applications. In analogy to somatostatin and gastrin-releasing peptide receptor-based radionuclide imaging, the current investigation confirms the promising performance of receptor antagonists for diagnostic use and potential further therapeutic application.

\section{CONCLUSION}

The high tumor accumulation and low uptake in normal tissues of ${ }^{111}$ In-3BP-227 warrants its further preclinical and ultimately clinical evaluation as an NTR1-targeting imaging agent. Moreover, the DOTA moiety of 3BP-227 allows the investigation of its therapeutic potential by labeling with ${ }^{177} \mathrm{Lu}$ or ${ }^{90} \mathrm{Y}$ without the need for additional structural modifications, which is currently under way in our laboratories.

\section{DISCLOSURE}

The costs of publication of this article were defrayed in part by the payment of page charges. Therefore, and solely to indicate this fact, this article is hereby marked "advertisement" in accordance with 18 USC section 1734. This work was supported by the KMU Innovativ program of the German Federal Ministry of Education and Research (BMBF, grant 0315865) and the Foundation of German Business (SDW). Frank Osterkamp, Ulrich Reineke and Christiane Smerling are cofounders, shareholders, and employees of 3B Pharmaceuticals $\mathrm{GmbH}$ and named inventors of the described compounds. No other potential conflict of interest relevant to this article was reported.

\section{ACKNOWLEDGMENTS}

We thank Franziska Stoeber for her help with analyzing the data, Ines Heinemann for her expert technical assistance, and Aileen Hoehne for critical review of the manuscript.

\section{REFERENCES}

1. Reubi JC. Peptide receptors as molecular targets for cancer diagnosis and therapy. Endocr Rev. 2003;24:389-427.

2. Körner M, Waser B, Strobel O, Büchler M, Reubi JC. Neurotensin receptors in pancreatic ductal carcinomas. EJNMMI Res. 2015;5:17.

3. Wu Z, Martinez-Fong D, Trédaniel J, Forgez P. Neurotensin and its high affinity receptor 1 as a potential pharmacological target in cancer therapy. Front Endocrinol (Lausanne). 2013;3:184.

4. Dupouy S, Mourra N, van Doan K, Gompel A, Alifano M, Forgez P. The potential use of the neurotensin high affinity receptor 1 as a biomarker for cancer progression and as a component of personalized medicine in selective cancers. Biochimie. 2011;93:1369-1378.

5. Morgat C, Mishra AK, Varshney R, Allard M, Fernandez P, Hindié E. Targeting neuropeptide receptors for cancer imaging and therapy: perspectives with bombesin, neurotensin, and neuropeptide-Y receptors. J Nucl Med. 2014;55:1650-1657.

6. Dash A, Chakraborty S, Pillai MR, Knapp FF Jr. Peptide receptor radionuclide therapy: an overview. Cancer Biother Radiopharm. 2015;30:47-71.

7. Wild D, Fani M, Behe M, et al. First clinical evidence that imaging with somatostatin receptor antagonists is feasible. J Nucl Med. 2011;52:1412-1417.

8. Cescato R, Maina T, Nock B, et al. Bombesin receptor antagonists may be preferable to agonists for tumor targeting. J Nucl Med. 2008;49:318-326.

9. Gully D, Labeeuw B, Boigegrain R, et al. Biochemical and pharmacological activities of SR 142948A, a new potent neurotensin receptor antagonist. J Pharmacol Exp Ther. 1997;280:802-812.

10. Gully D, Canton M, Boigegrain R, et al. Biochemical and pharmacological profile of a potent and selective nonpeptide antagonist of the neurotensin receptor. Proc Natl Acad Sci USA. 1993;90:65-69.

11. Osterkamp F, Smerling C, Reineke U, Haase C, Ungewiss J. inventors; 3BP Pharmaceuticals $\mathrm{GmbH}$, assignee. Neurotensin receptor ligands. European patent application EP20120008208. December 7, 2012.

12. Vita $\mathrm{N}$, Laurent $\mathrm{P}$, Lefort $\mathrm{S}$, et al. Cloning and expression of a complementary DNA encoding a high affinity human neurotensin receptor. FEBS Lett. 1993;317:139-142.

13. Botto JM, Chabry J, Sarret P, Vincent JP, Mazella J. Stable expression of the mouse levocabastine-sensitive neurotensin receptor in HEK 293 cell line: binding properties, photoaffinity labeling, and internalization mechanism. Biochem Biophys Res Commun. 1998;243:585-590.

14. Lang C, Maschauer S, Hübner H, Gmeiner P, Prante O. Synthesis and evaluation of a ${ }^{18} \mathrm{~F}$-labeled diarylpyrazole glycoconjugate for the imaging of NTS1-positive tumors. J Med Chem. 2013;56:9361-9365. 